\title{
Article \\ Protocol of Action in Injuries of the Long Head of the Biceps Tendon. Joint Reference Values and Recommended Training Weight for the Recovery of Joint Range and Strength
}

\author{
Sagrario Pérez-de la Cruz
}

check for

updates

Citation: Pérez-de la Cruz, S. Protocol of Action in Injuries of the Long Head of the Biceps Tendon.

Joint Reference Values and

Recommended Training Weight for the Recovery of Joint Range and Strength. Appl. Sci. 2021, 11, 10382. https://doi.org/10.3390/ app112110382

Academic Editor: Johann Zwirner

Received: 21 October 2021

Accepted: 3 November 2021

Published: 5 November 2021

Publisher's Note: MDPI stays neutral with regard to jurisdictional claims in published maps and institutional affiliations.

Copyright: (C) 2021 by the author. Licensee MDPI, Basel, Switzerland. This article is an open access article distributed under the terms and conditions of the Creative Commons Attribution (CC BY) license (https:// creativecommons.org/licenses/by/ $4.0 /)$.
Department of Nursing, Physical Therapy and Medicine, University of Almería, La Cañada de San Urbano, 04120 Almería, Spain; spd205@ual.es; Tel.: +34-950214574

\begin{abstract}
Daily and sports activities can be affected by joint injury. The objectives of physical therapy are to help patients regain range of motion (ROM), mobility, strength, and function. The purpose of this study was to examine the optimal joint range of elbow flexion and recommended training weight for the recovery of biceps brachii muscle strength. It was a prospective study with a sample of 157 healthy young adult volunteers from two institutions. The Hawk goniometer was used for measurements. Participants performed a flexion-extension movement of the elbow in standing, in series, with progressive loads. The ROM in males was found to be higher than in females. Nevertheless, the joint values of the dominant and non-dominant arm indicate that there are differences between both arms, regardless of gender. In relation to the force used during the performance of the activity, the weight used by the women was lower than that of the men, whereas, during the execution, women showed greater stability throughout the test, compared to men. Normative values have been established for the ROM and weights to be used in the recovery process of injuries to the long tendon of the biceps brachii muscle.
\end{abstract}

Keywords: range of motion; rehabilitation; elbow; strength; weight; biceps brachii muscle

\section{Introduction}

Musculoskeletal pathologies of the shoulder-elbow joints represent a significant cause of morbidity [1]. In primary care in Spain, this problem affects one fifth of the population and increases with age, reaching its highest prevalence in the 50 to 59 age group (25.9\% of the total population) [2]. Among the most frequently encountered injuries in clinical practice, lesions of the long head of the biceps tendon are one of the most frequent causes of shoulder pain and functional impairment in young adults [3-6]. This tendon is often involved in shoulder pathologies and can present clinically relevant ailments along its entire course, from the musculotendinous junction to its origin on the glenoid labrum [3]. These injuries can have a significant effect on health and other aspects such as sports, together with social and/or occupational activities. Moreover, they represent an increased cost for the healthcare system compared to the rest of common pathologies due to the period of incapacity for work that this entails for the person [2]. Recovery of shoulder and elbow function after conservative or surgical treatment is essential for patients and the preservation of functional independence. Further research is necessary in terms of range of motion (ROM) references and measurement of execution speed to prevent overuse injuries and support the use of therapy aimed at stabilizing movement along the entire joint pathway (functional and final joint range). For example, after a period of immobilization following surgery or rest, these aspects are important in common clinical practice to recover strength to optimal levels [7-9].

For the measurement of ROM, joint range is usually compared with the contralateral side or with a group of individuals with similar characteristics, assuming that the uninjured side can be used as an appropriate reference [10]. Generally, examinations and assessments 
after any intervention are targeted to the achievement of an objective and reliable recovery of muscle function. It is, therefore, difficult to recommend a particular muscle recovery and strengthening approach without a quantification of the potential deficit in biceps brachii muscle function [11,12]. The literature shows that the anthropometric factors of patients must be considered to achieve an optimal recovery (age, activity level and sex) [12], however, these data are currently difficult to quantify and obtain. Morrey et al. stated that the functional ROM of the elbow for daily activity was $0-130^{\circ}$ flexion-extension and $50^{\circ}$ pronation-supination in any direction [13], although there is no unanimity among authors on this point (due to new specific contemporary tasks in daily life, which entail a modification of these standard values) [14-16].

Prevention is a key factor for reducing the chances of injury. Adequate progression, intensity, frequency, and load can cause a decrease in these possibilities, especially in the case of overload injuries. Hence the importance of identifying risk factors of recurrence in injuries of the long head of the biceps tendon in order to design prevention, health promotion and recovery programs for this type of injury [1]. Although prevention has always been a subject of discussion, very little progress has been made in this field. Indeed, normative data for ROM in the population, according to their physical activity are necessary as there is a lack of objective data [17]. Considering that a greater number of studies on shoulder-elbow injuries due to injury to the biceps brachii longus tendon and ranges of motion and speed in sports practice are needed, the number of studies focused on clinical practice is even more limited. Moreover, the number of studies related to the field of clinical rehabilitation (and not sport) is even smaller. Therefore, it is necessary to propose a line of work to determine which values would be adequate for the recovery of joint mobility and strength recovery with the use of resistance training.

The aim of the present study was to examine the standard joint range of elbow flexion and recommended training weight for the recovery of biceps brachii muscle strength in a population of healthy subjects without a history of injury to the long portion of the biceps tendon. Furthermore, this study sought to analyze the possible anthropometric influencing factors in order to define predictive values for young adults.

\section{Materials and Methods}

\subsection{Subjects}

A total of 157 students (81 men and 76 women, with an age range of 21 to 34 years), from a National Police School and the Faculty of Health Sciences of the University of Almería (Almería, Spain) volunteered to participate in this study. All participants were asked to sign the informed consent form prior to their participation in the study. Ethical approval for this study was granted by the Bioethics Committee of the University of Almería (Almería, Spain) (Ref: UALBIO2020/022), with the authorization of the Director of the Police School and the Physical Culture Department. This study was conducted between January and February 2020. The inclusion criteria were people over 18 years of age, with the ability to cooperate with verbal commands and motor actions requested during the test, and with no history of surgery, trauma, or pain during the last six months in the shoulder joint (left and right), and whose dominant arm was the right arm (with the left arm being the non-dominant arm). The exclusion criteria were as follows: a deterioration of health status or general condition, joint limitation of the upper limb (shoulder-elbow-hand), pain during the test, history of traumatic or neurological event, or inflammation of the requested joint in the last six months.

\subsection{Measurement Instrument}

Measurements were made using the Hawk goniometer. This plastic device, rectangular in shape and measuring $43 \times 38 \times 17 \mathrm{~mm}$, records the angular movement when the joint to be measured moves in any of the three coordinate axes ( $\mathrm{X}, \mathrm{Y}$ and $\mathrm{Z})$. If the segment to be measured moves in an angular movement, it captures the angle using an internal gyroscope. The device must always be positioned in the same arrangement so that the 
axes of the device are the same. It is not necessary to always place the device at the same distance from the joint, since the measurement is angular, and therefore, the data are the same, independent of the lever arm. This measurement is sent through a Bluetooth system to a mobile phone that has previously downloaded the application designed by the manufacturer of the device (Human Computer Technologies-HCT company, Hong Kong, China). The phone screen then displays the three angles of the movement performed, and the angle in relation to the $\mathrm{X}$ axis, $\mathrm{Y}$ axis and $\mathrm{Z}$ axis. This device has a measurement range of $360^{\circ}$, based on a scale of $1^{\circ}$ increments $[18,19]$. An intraclass correlation coefficient (ICC) of above 0.9 was obtained, indicating a high intra-evaluator reliability of the Hawk goniometer [19].

\subsection{Procedure}

Before commencing the data collection and evaluation process, the study procedure was explained to the study participants and any questions related to the study were answered. After applying the inclusion and exclusion criteria, the procedure to be followed was further explained to those admitted. The weight, height, and body mass index of each of the participating volunteers were recorded. Information was collected on a personal data sheet and assessment of joint range was collected with the use of the sensor. The approximate duration of the entire process was thirty minutes.

The measurements and appointments were randomly assigned to avoid possible evaluator bias. The participants were asked to perform the test with the limb to be evaluated unclothed, to avoid any restriction of the requested movement and to allow the active movement to be performed following the instructions given by the evaluator. For the measurement of the selected elbow movement, the individuals were placed in a standing position, with the trunk supported on a surface to allow free movement of both arms during the measurement of each side, and the trunk area stabilized to avoid possible compensations. The system detects any indication that may suggest that the person is not performing the action in a stable manner (precision in the performance of the movement, which means not performing muscle compensations and performing the movement in a single movement plane). Regarding the placement of the Hawk system, the sensor was placed on the anterior aspect of the wrist, where it would not interfere with clothing or any other type of device. The system was held in place by a strap and the goniometer was placed inside the strap (Figure 1). The only important consideration when measuring and recording data was to ensure that the device did not move from its initial location, thanks to the proper adhesion of the strap. When the device receives the data, it indicates whether there has been any type of alteration in the analyzed movement, which would reveal whether it was valid or not.

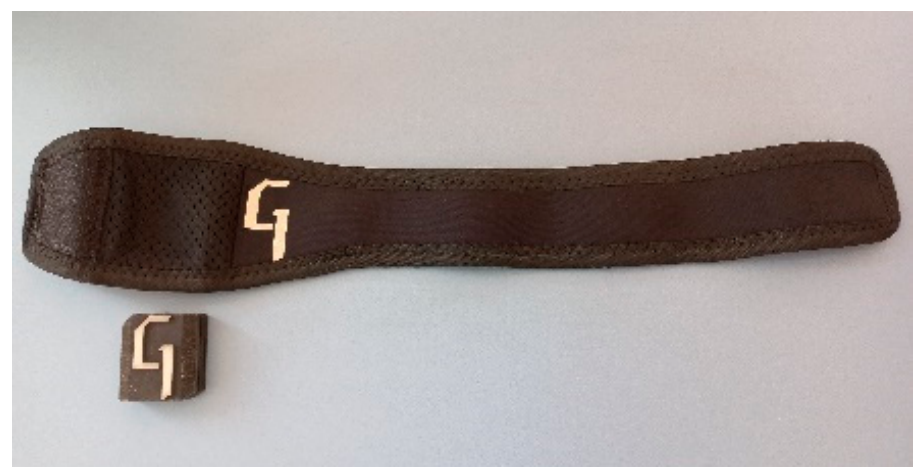

Figure 1. Hawk goniometer.

Both elbows (dominant and non-dominant upper limb) were tested in the same position and joint range. Each participant was allowed three submaximal trials prior to data collection, to ensure familiarization with the system and test procedure. The protocol consisted of performing five repetitions of elbow flexion at an angular velocity of 
$60^{\circ} / \mathrm{s}$, allowing a one-minute rest, followed by another five repetitions at a higher velocity $\left(180^{\circ} / \mathrm{s}\right)$, controlled by a device's own isokinetic system. A rest period was granted between the measurements of both arms. Each of the series was evaluated with progressive loads, to evaluate the flexion ROM and speed with different loads (when recovering strength after an injury, work with progressive loads is proposed, according to the patient's tolerance). The entire process was supervised by a physiotherapist with expertise in the evaluation process with the measurement system used. This protocol is the one followed by related studies, such as the study by Samah Mamoud with a population of children and adolescents [18].

\subsection{Statistical Analysis}

The data analysis was performed using the Statistical Package for Social Science (SPSS) software version 25 for Windows. A Kolmogorov-Smirnov test with a 95\% confidence interval (CI) was performed to establish the normality of continuous data distributions. The Pearson correlation coefficient evaluated the possible associations between age, level of physical activity, BMI, and ROM, and data were analyzed by use of an unpaired $t$ test to assess differences between sexes. A $p$ value of $<0.05$ was considered significant.

\section{Results}

The final study sample included 157 students, of whom 81 (51.6\%) were men and 76 were women (48.4\%) ranging in age from 21 to 34 years and with a mean age of 28.92 years (SD 2.7). Regarding hand dominance, the total sample was right-handed. Table 1 shows the socio-demographic data of the sample.

Table 1. Socio-demographic data of the sample.

\begin{tabular}{cccc}
\hline & Min-Max & Mean \pm SD & Women (Mean \pm SD)/Men (Mean \pm SD) \\
\hline Age & $21-38$ & $28.92 \pm 2.7$ & $29.62 \pm 2.47 / 32.63 \pm 4.91$ \\
\hline Height $(\mathrm{cm})$ & $162-194$ & $175.26 \pm 5.61$ & $170.28 \pm 4.60 / 174.33 \pm 2.78$ \\
\hline Weight $(\mathrm{kg})$ & $52-93$ & $74.19 \pm 8.11$ & $68.46 \pm 6.01 / 77.81 \pm 5.05$ \\
\hline Physical activity (hours/week) & $4-18$ & $7.83 \pm 1.94$ & $6.79 \pm 0.49 / 9.25 \pm 1.16$ \\
\hline BMI & $18.2-29.2$ & $23.45 \pm 3.19$ & $21.33 \pm 2.08 / 26.28 \pm 3.04$ \\
\hline
\end{tabular}

BMI: body mass index//cm: centimeters//SD: standard deviation.

Table 2 shows the results obtained between flexion of the right arm and of the left arm. Measurements were first made without weight, subsequently the weight progressively increased until reaching the individual's maximum weight. The flexion of the dominant side in males was $115.56 \pm 15.71$ degrees, whereas the flexion described for the female sex and dominant arm was $118.42 \pm 11.61$. There were no statistically significant differences between the two measurements (measurements made on the dominant side in both sexes) $(p=0.319)$. In relation to the non-dominant arm, the values shown by the male sample were $112.87 \pm 9.50$ degrees, whereas in the women the values were $108.63 \pm 10.18$ degrees. In this case, there were also no statistically significant differences between the values shown by the two variables $(p=0.204)$. Observing the results obtained, there were no statistically significant differences in the range of motion of dominant and non-dominant upper limbs of men and women.

The weights used to perform the elbow flexion-extension movement in degrees of functionality varied according to the gender of the participants (Figures 2 and 3). In the case of males, the weights $\left(\mathrm{kg}, x\right.$-axis) and velocity $\left(\mathrm{mm} / \mathrm{s}^{2}, y\right.$-axis) used were graded up to eight kilograms (for males belonging to the younger age group), whereas in the case of women, they were unable to perform the action with this weight, and the weight decreased (maximum six kilograms, with part of the female sample not being able to reach this weight). In both groups, the younger participants demonstrated greater stability (precision in the performance of the movement, which means not performing muscle compensations) 
in the execution of the gesture than the older volunteers. According to gender, the women displayed greater stability throughout the functional ROM compared to the men, who needed more weight to stabilize the entire movement requested.

Table 2. ROM of the right and left arm.

\begin{tabular}{lccc}
\hline \multirow{2}{*}{ ROM Right Arm } & Total Mean (DT) & \multicolumn{2}{c}{ Gender, Mean (SD) } \\
\cline { 3 - 4 } & & Female & Male \\
\hline ROM_0 kg & $116.8-162.26$ & $116.8-162.26$ & $116.8-157.07$ \\
ROM_1 kg & $117.27-157.4$ & $120.85-156.32$ & $117.27-157.4$ \\
ROM_2 kg & $106.18-159.39$ & $116.5-159.33$ & $106.18-159.39$ \\
ROM_3 kg & $100.93-167.05$ & $119.26-157.01$ & $100.93-167.05$ \\
ROM_4 kg & $102.06-164.14$ & $114.37-152.97$ & $102.06-164.14$ \\
ROM_5 kg & $99.5-157.25$ & $113.16-153.37$ & $99.5-157.25$ \\
ROM_6 kg & $103.29-150.59$ & $111.12-147.87$ & $103.29-150.59$ \\
ROM_7 kg & $103.71-155.73$ & $126.5-153.54$ & $103.71-155.73$ \\
ROM_8 kg & $99.13-140.72$ & - & $99.13-140.72$ \\
\hline & & \multicolumn{2}{c}{ Gender, Mean (SD) } \\
ROM Left Arm & Total Mean (DT) & Female & Male \\
\cline { 3 - 4 } & & $118.22-159.87$ & $104.74-161.89$ \\
ROM_0 kg & $104.74-161.89$ & $124.91-160.14$ & $115.19-160.6$ \\
ROM_1 kg & $115.19-160.6$ & $112.39-160.13$ & $112.39-162.1$ \\
ROM_2 kg & $112.39-162.1$ & $98.8-156$ & $98.8-159.09$ \\
ROM_3 kg & $98.8-159.09$ & $94.32-155.43$ & $94.32-155.8$ \\
ROM_4 kg & $94.32-155.8$ & $107.94-149.01$ & $100.77-152.08$ \\
ROM_5 kg & $100.77-152.08$ & $105.46-144.47$ & $102.82-158.02$ \\
ROM_6 kg & $102.82-158.02$ & $138.11-152.27$ & $98.09-152.27$ \\
ROM_7 kg & $98.09-152.27$ & - & $113.26-118.15$ \\
ROM_8 kg & $113.26-118.15$ & &
\end{tabular}
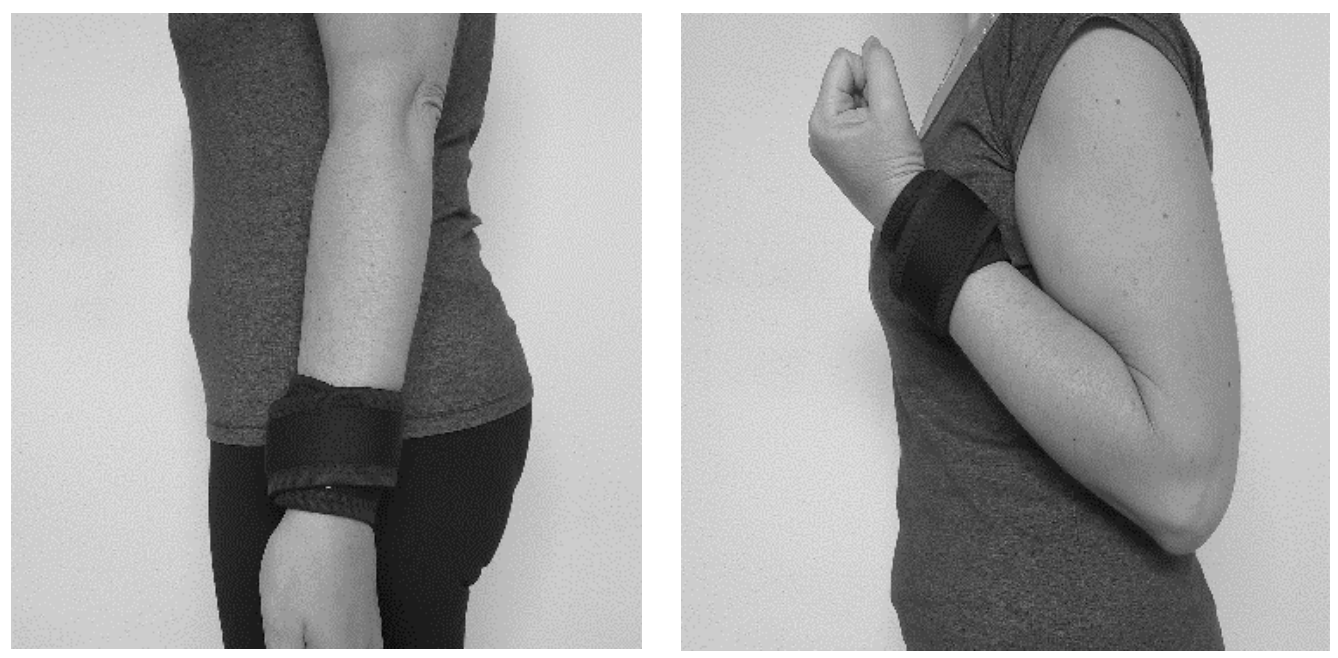

Figure 2. Sensor placement and movement tested. 


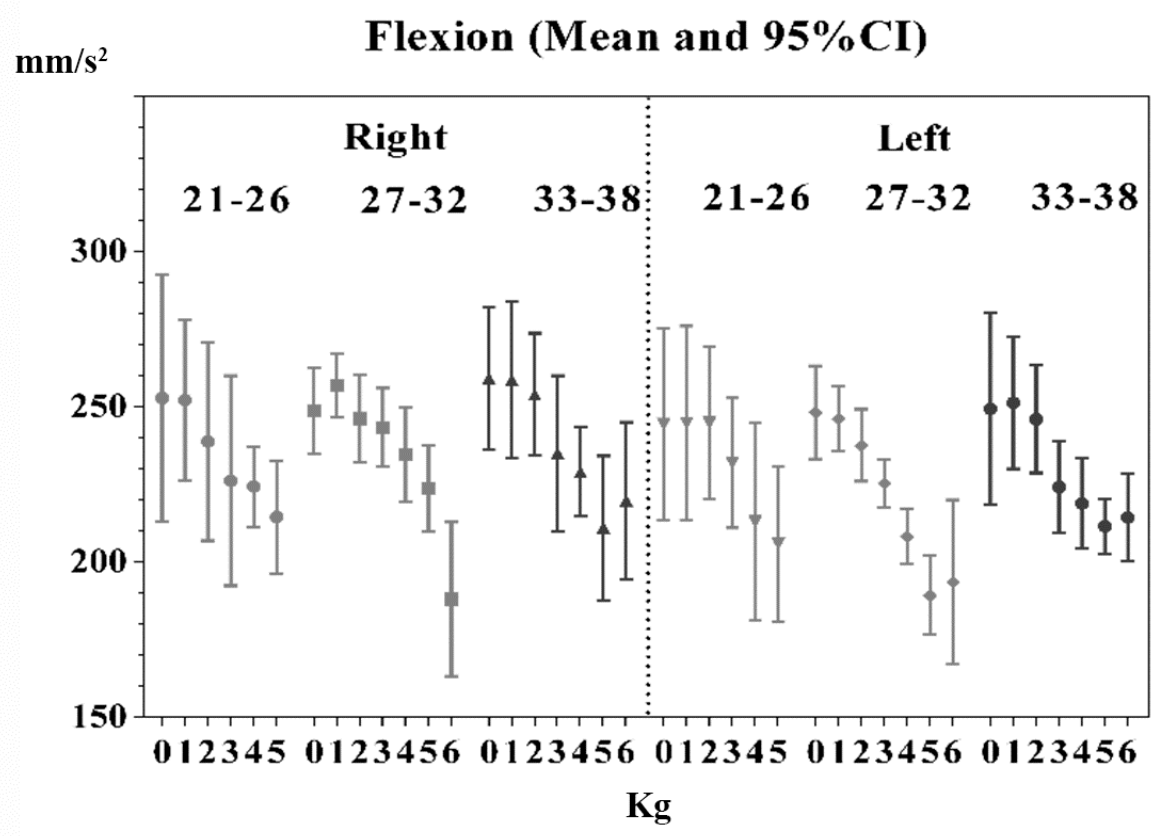

(A)

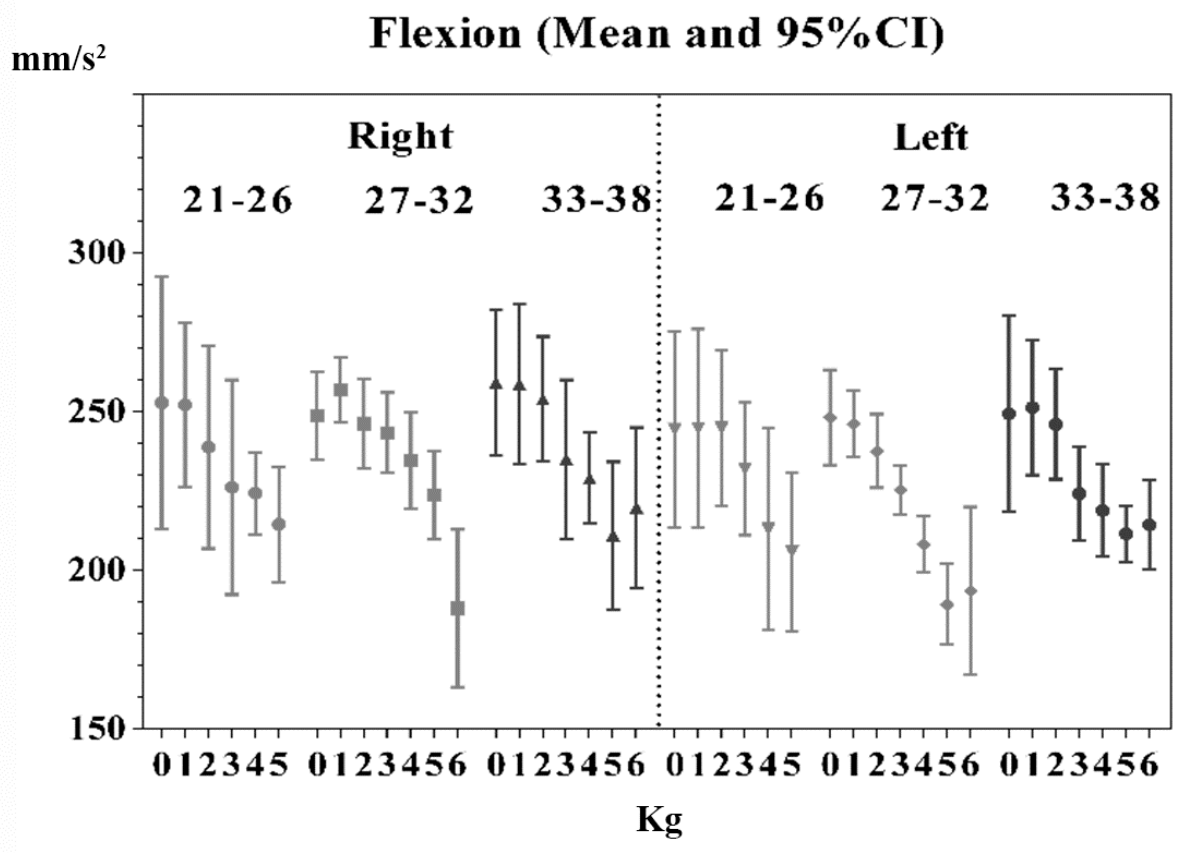

(B)

Figure 3. (A): Speed-weight relationship for the movement of flexion in males. (B): Speed-weight relationship for the push-up movement in women.

Figure 3 show the speeds obtained in the execution of the test with different weights by the volunteers, while Figure 4 show the extension speed. 


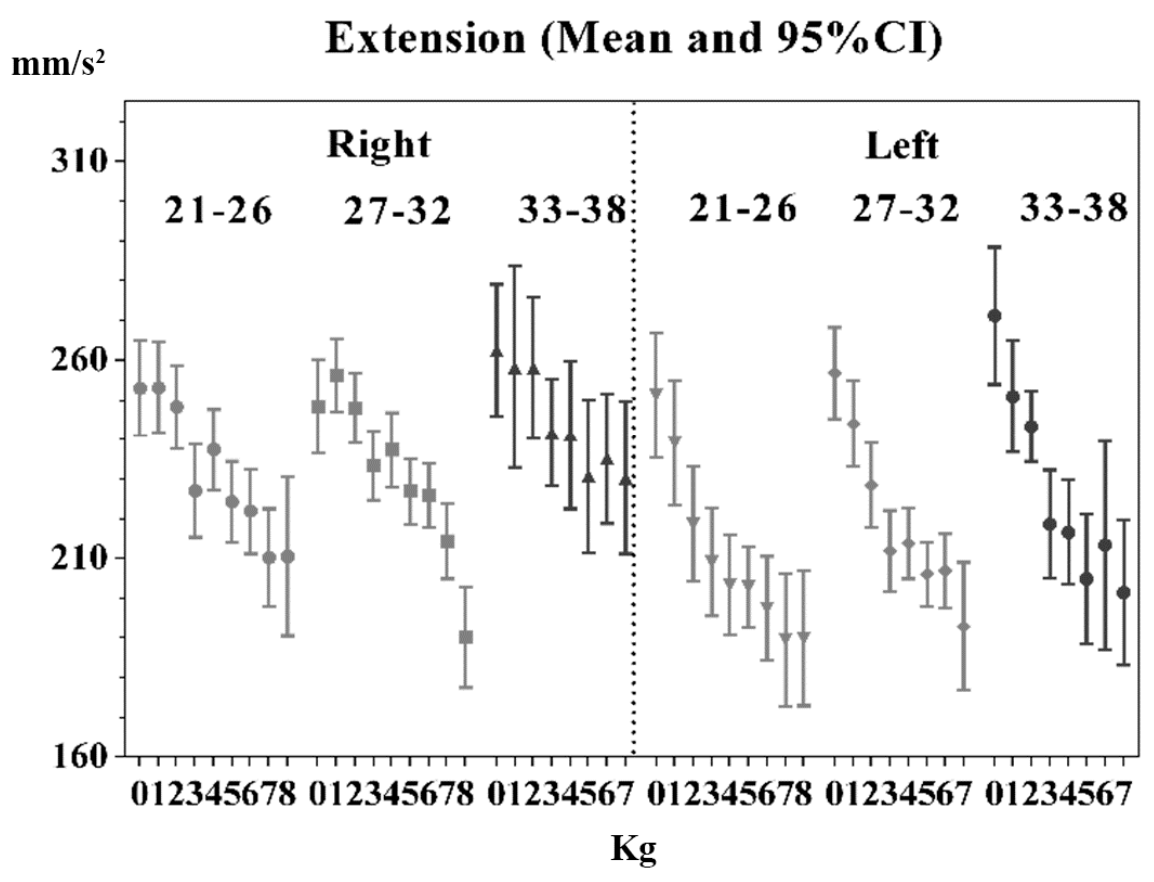

(A)

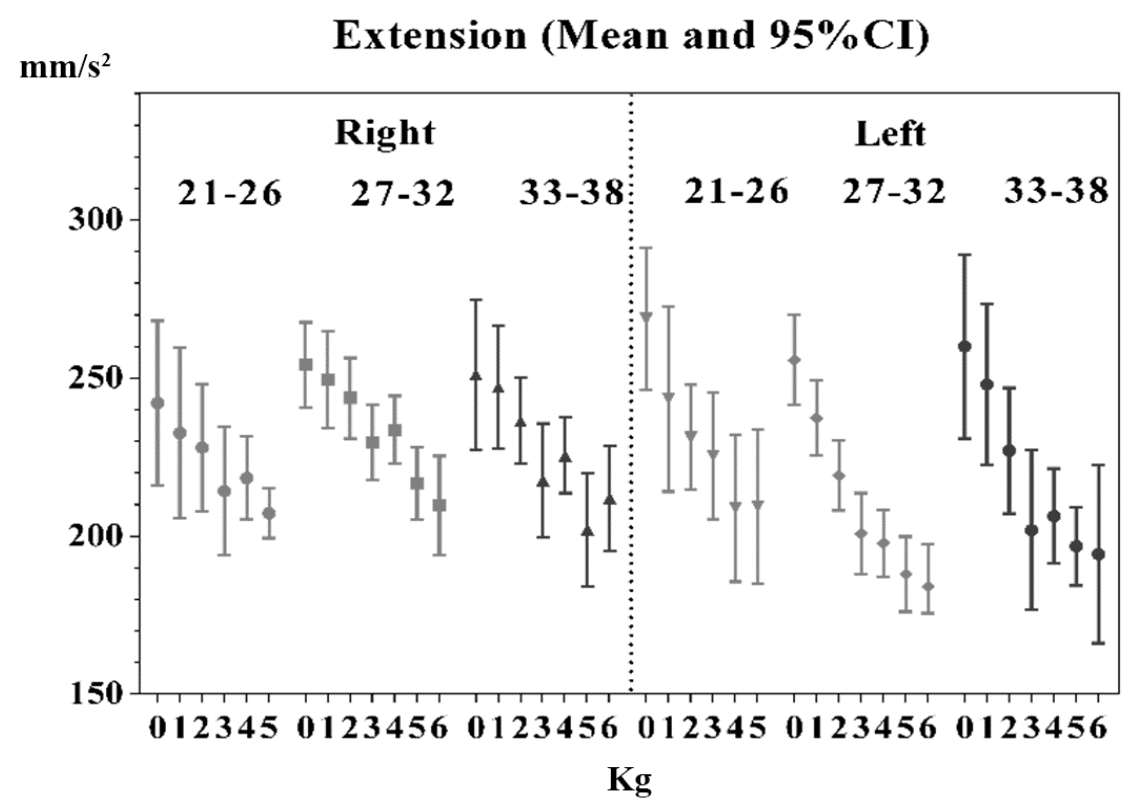

(B)

Figure 4. (A): Speed-weight relationship in movement extension in males. (B): Speed-weight relationship in the extension movement in women.

\section{Discussion}

In today's clinical practice, professionals have accurate mobile devices at their disposal to improve their assessments. Isokinetic devices enable practitioners to assess muscle strength and determine which muscle group requires rehabilitation after an injury, as well as predicting joint stability. The advantages, indications and efficacy of diagnostic testing and exercise have been well documented $[7,13,14,19]$.

To assess muscle strength, references are generally taken from both sides (dominant and non-dominant sides are included) with an assumption of bilateral equivalence. Asym- 
metry between equivalent body sides may indicate greater pathology and dysfunctional kinematics [20]. The augmented average amount of soft tissue (i.e., muscle) could restrict range of motion without influencing the straightened elbow. Some studies suggest that laterality could influence the overall upper extremity ROM. For example, the effect of dominance on upper arm ROM was observed in certain movements, some of which increased on the dominant side, whereas others were decreased $[12,18,20]$.

Therefore, it is important to detect asymmetry in functional movements during the initial stage of rehabilitation. Most of the population has a preferred or dominant side. The dominant side is generally used over the non-dominant side during most functional movements, including physical activity. The study by Mehmet F. Güleçyüz et al. compared the dynamic kinematics of the upper limbs during elbow flexion movements (biceps brachii action) with free weights on each arm in healthy young right-handed adults [20]. The greatest measured effect of the biceps brachii in supination was observed with the elbow flexed at $90^{\circ}$ and the forearm in neutral or a slightly supinated position. In contrast, the lowest impact was recorded with maximum extension at the elbow and the forearm in pronation $[20,21]$. Hence, the determination of the starting position and gesture to be evaluated.

\subsection{Range of Motion of the Elbow Joint}

There were no significant differences in joint range between sexes. The findings of this paper contrast with the findings of a previous study by Chapleau et al. [22], where no correlation was found between the level of physical activity or laterality and changes in elbow mobility. Women presented a greater degree of flexion and total ROM of the elbow than men. This study also confirms the association between several demographic and anthropometric factors and elbow ROM in healthy adults. Among these, the body mass index and forearm circumference are mainly responsible for the variations in mobility among the population. In our case, the results show that gender has an influence, albeit not significant, on the ROM of the elbow joint, involving the action of the long tendon of the biceps due to its flexor action of the joint. Our study coincides with Chapleau's study by obtaining the largest joint ranges in males, with a more voluminous muscle mass than the female sample.

It is also interesting to comment on the results obtained by the sample of young adults used in the study by Valone et al. [23], with a sample of adolescents and children. The mean ROM was 28 to 146 degrees of elbow flexion/extension and 40 to 148 degrees of joint amplitude for the performance of activities of daily living. In our study, the ROM decreases as the weight used in the evaluation test increases, however, we can affirm that it is still within the range considered functional, although it does not fall within the age range studied by Valone [23]. Joint amplitude is influenced by age, decreasing as the age of the sample increases (comparing the values of the study conducted in children and our sample of young adults). However, these changes are not considered significant, providing no factors are present that may accentuate these differences (such as sports lesions).

Our sample included adults who played amateur sports, raising a theory that may be in part corroborated by these results. One of the reasons why a young adult may not reach these ranges of motion may be due to adaptive changes caused by repetitive microtrauma that occurs in adolescence (early formative career years for many athletes) or due to pre-existing inherited differences [19,24,25].

This study proposes gender-specific reference values for elbow joint range for healthy young adults, which may serve as a reference for the recovery of joint range after a traumatic event. These reference values can be used for future related research.

\subsection{Strength and Speed of Movement Execution}

The application of force in the recovery of biceps brachii longus tendon injuries is a risk factor for injury or recurrence in patients. In this study we sought to determine the optimal framework for introducing an adequate treatment and/or injury prevention program on 
this anatomical structure. The sample was a population of young, healthy adults, who were not at risk of injury, contributing to a better understanding of normality data to provide data on when adaptations to exertion begin to occur. This study suggests that gender and age, as well as upper limb dominance, may guide therapists' clinical practice decisions. The data have shown that women and the non-dominant side displays weakness compared to males and the dominant side, therefore, the performance of strengthening programs is recommended to minimize the risk of injury, as concluded in other studies related to sport and injury prevalence $[26,27]$.

The flexion strength of males was significantly higher than that of the female group. The values obtained in this study were higher than the values reported in other experimental setups [16,24]. Throughout the age range analyzed in our sample, the dominant and contralateral limb can be used as a good reference by the clinician. The statement by Shank et al. [28] that the dominant arm results in significantly greater flexion, indicates that this fact should be considered when proposing specific motor activities for each gender and the injured upper limb.

In relation to the speed of execution of the exercise, a greater control and precision of the gesture on the dominant side was observed thanks to comparisons of angular velocity. In the male participants, the execution speeds in the concentric movement were quite similar, becoming more amplified when using the maximum weight and in patients in the sample who were older. In the case of the women, however, bearing in mind that the maximum weight supported in the test was lower (six kilos), the flexion movement of the dominant and non-dominant arm in the younger age range was more dispersed, compared to that of the men. Whereas in the women with a higher age range, the non-dominant arm was more stable in terms of the execution speed than the dominant arm, which implies that working with weights close to the limit shown in the study (seven kilos in men and six kilos in women) means that the speed in the execution of the motor act is lower (i.e., a slower movement), with greater joint stability and no muscular compensations that could alter the specific work of the injured muscle.

Regarding the eccentric muscle action, both in men and women of the sample up to the age of 32 years, similar results were obtained, however, in the non-dominant arm, both in men and women, the results were more extensive.

Overall, the speed and accuracy (lack of compensation) of the dominant sides were superior to the non-dominant sides in the subjects evaluated [25]. The results could be interpreted as indicating that the dominant sides were able to sacrifice and partially control the maximum potential velocity to improve movement accuracy [25]. Therefore, dominant arms were able to control the degree of acceleration and deceleration more precisely for optimal control of limb movement compared to the contralateral side. Non-dominant sides have been found to have less strength, power, refined motor control and maximal velocity $[25,26]$. Although lateral arm raises are easy to perform, the movement involves a complex coordination of several areas of the body, including muscles, ligaments and tendons around the trunk, scapula, shoulders, elbows, and wrists $[27,29]$. The central body segment and lower extremities are also involved in stability and isometric control during upper limb movements that involve working with or against gravity $[27,29-33]$. It is widely known that most athletes and physical training participants experience asymmetry in movement and musculoskeletal impairment due to specific body segments used repeatedly $[2,3,9,25]$. According to the results obtained in this study, some asymmetry is perceived, although not as pronounced as that shown by professional athletes. This may be due to the fact that our sample is not dedicated to professional sports, and therefore, they do not develop such pronounced asymmetries. However, due to the repeated dominant use of the preferred sides without adequate compensatory intervention, many individuals acquire muscle imbalances or asymmetries at an early age $[3,30]$. Therefore, early detection and observation to promote the correction of muscle imbalance and asymmetry in movement is recommended, together with the prescription of corrective interventions. 
Regarding the limitations of this study, the age range is limited to young adults, however it could be extended to continue analyzing the motor behavior of populations that are candidates to suffer some type of pathology in the upper limb. More specifically, it would be interesting to focus the study on professions or people whose work/sports dedication is highly demanding for their upper limbs, to corroborate or challenge these findings. The sample size should be increased to increase the reliability of the results obtained.

\section{Conclusions}

Specific differences in movement patterns were observed between dominant and non-dominant arms during movements involving the motor action of the long tendon of the biceps brachii muscle. The dominant limbs were able to control the ROM and concentric and eccentric velocity during the entire joint path and with the incorporation of weights in the execution of the motor action, compared to non-dominant sides. Furthermore, a characteristic behavior was observed according to the sex of the patient.

Certain aspects should be considered, such as joint amplitude, where the functional ranges vary according to the patient's gender, although not significantly, and strength and speed in the execution of the movement, with the results showing that the female gender and non-dominant sides present weakness compared to the male gender and dominant sides. According to the results of this study, weights in excess of eight kilograms in men and seven kilograms in women are not recommended when it comes to recovering strength, as they can cause damage to the joint and exceed the subjects' functional capacity.

Funding: This research received no external funding.

Institutional Review Board Statement: The study was conducted according to the Declaration of Helsinki guidelines and was approved by the University of Almería's Institutional Review Board (or ethics committee) (UALBIO2020/022).

Informed Consent Statement: Informed consent was obtained from all subjects involved in the study.

Acknowledgments: The author wishes to thank the National Police School's Department of Physical Education in Ávila, Spain, especially Óscar Arellano y Antonio Vargas for their participation in this study, and thanks to A.F.B. for helping with statistical analysis.

Conflicts of Interest: The author has declared no conflict of interest.

\section{References}

1. Zaremski, J.L.; Galloza, J.; Sepulveda, F.; Vasilopoulos, T.; Micheo, W.; Herman, D.C. Recurrence and return to play after shoulder instability events in young and adolescent athletes: A systematic review and meta-analysis. Br. J. Sports Med. 2017, 51, 177-184. [CrossRef]

2. Vicente-Pardo, J.M. Hombro doloroso e incapacidad temporal. El retorno al trabajo tras larga baja por hombro doloroso. Causalidad del trabajo en el hombro doloroso. Med. Segur. Trab. 2016, 62, 337-359.

3. Ge, H.; Zhang, Q.; Sun, Y.; Li, J.; Sun, L.; Cheng, B. Tenotomy or tenodesis for the long head of biceps lesions in shoulders: A systematic review and meta-analysis. PLOS ONE 2015, 10, e0121286. [CrossRef]

4. Khazzam, M.; George, M.S.; Churchill, R.S.; Kuhn, J.E. Disorders of the long head of biceps tendón. J. Shoulder Elb. Surg. 2012, 21, 136-145. [CrossRef]

5. Urita, A.; Funakoshi, T.; Amano, T.; Matsui, Y.; Kawamura, D.; Kameda, Y.; Iwasaki, N. Predictive factors of long head of the biceps tendon disorders-the bicipital groove morphology and subscapularis tendon tear. J. Shoulder Elb. Surg. 2016, 25, 384-389. [CrossRef]

6. Refior, H.J.; Sowa, D. Long tendon of the biceps brachii: Sites of predilection for degenerative lesions. J. Shoulder Elb. Surg. 1995, 4, 436-440. [CrossRef]

7. Abrams, G.D.; Safran, M.R. Diagnosis and management of superior labrum anterior posterior lesions in overhead athletes. Br. J. Sports Med. 2010, 44, 311-318. [CrossRef]

8. Pinto-Carral, A.; Fernández Villa, T.; Molina de la Torre, A. Patient reported mobility: A systematic review. Arch. Phys. Med. Rehabil. 2016, 97, 1182-1194. [CrossRef] [PubMed]

9. Schultz, J. Clinical evaluation of the shoulder. Phys. Med. Rehabil. Clin. N. Am. 2004, 15, 351-371. [CrossRef] [PubMed]

10. Hsu, A.R.; Ghodadra, N.S.; Provencher, C.M.T.; Lewis, P.B.; Bach, B.R. Biceps tenotomy versus tenodesis: A review of clinical outcomes and biomechanical results. J. Shoulder Elb. Surg. 2011, 20, 326-332. [CrossRef] 
11. Frost, A.; Zafar, M.S.; Maffulli, N. Tenotomy versus tenodesis in themanagement of pathologic lesions of the tendón of the long head of the biceps brachii. Am. J. Sports Med. 2009, 37, 828-833. [CrossRef] [PubMed]

12. Friedman, J.L.; FitzPatrick, J.L.; Rylander, L.S.; Bennett, C.; Vidal, A.F.; McCarty, E.C. Biceps tenotomy versus tenodesis in active patients younger than 55 years is there a difference in strength and outcomes? Orthop. J. Sports Med. 2015, 3, 1-6. [CrossRef] [PubMed]

13. Morrey, B.F.; Askew, L.J.; Chao, E.Y. A biomechanical study of normal functional elbow motion. J. Bone Jt. Surg. Am. 1981, 63, 872-877. [CrossRef]

14. Barad, J.H.K.; Rachel, S.; Ebramzadeh ESilva, M. Range of motion of the healthy pediatric elbow. J. Pediatr. Orthop. 2013, 22, 117-122. [CrossRef]

15. Schreiner, A.J.; Schweikardt, N.; Gühring, D.; Ahrend, M.D.; Döbele, S.; Ahmad, S.S.; Baumann, M.; Hirschmann, M.T.; Bozzi, F.; Ateschrang, A. Arthroscopic arthrolysis leads to improved range of motion and health-related quality of life in post-traumatic elbow stiffness. J Shoulder Elbow Surg. 2020, 29, 1538-1547. [CrossRef] [PubMed]

16. Sardelli, M.; Tashjian, R.Z.; MacWilliams, B.A. Functional elbow range of motion for contemporary tasks. J. Bone Jt. Surg. Am. 2011, 93, 471-477. [CrossRef]

17. Wilps, T.; Kaufmann, R.A.; Yamakawa, S.; Fowler, J.R. Elbow Biomechanics: Bony and Dynamic Stabilizers. J. Hand Surg. Am. 2020, 45, 528-535. [CrossRef] [PubMed]

18. Ahmed Sheha, S.M.; Rasmy Elserougy, H.; Gaid Esdhom, M. Difference in Isokinetic Strength of Shoulder Joint Muscles in Dominant versus Nondominant Upper Extremities in Children. J. Nurs. Health Sci. 2019, 8, 32-36.

19. Pérez-de la Cruz, S.; de León, Ó.A.; Mallada, N.P.; Rodríguez, A.V. Validity and intra-examiner reliability of the Hawk goniometer versus the universal goniometer for the measurement of range of motion of the glenohumeral joint. Med. Eng. Phys. 2021, 89, 7-11. [CrossRef]

20. Güleçyüz, M.F.; Pietschmann, M.F.; Michalski, S.; Eberhard, F.M.; Crispin, A.; Schröder, C.; Mittermüller, M.J.; Müller, P.E. Reference Values of Flexion and Supination in the Elbow Joint of a Cohort without Shoulder Pathologies. BioMed Res. Int. 2017, 2017, 1654796. [CrossRef]

21. O'Sullivan, L.W.; Gallwey, T.J. Upper-limb surface electromyography at maximum supination and pronation torques: The effect of elbow and forearm angle. J. Electromyogr. Kinesiol. 2002, 12, 275-285. [CrossRef]

22. Chapleau, J.; Canet, F.; Petit, Y.; Sandman, E.; Laflamme, G.-Y.; Rouleau, D.M. Demographic and anthropometric factors affecting elbow range of motion in healthy adults. J. Shoulder Elb. Surg. 2013, 22, 88-93. [CrossRef]

23. Valone, L.C.; Cameron, W.; Tartarilla, A.B.; Whited, A.; Sugimoto, D.; Bae, D.S.; Bauer, A.S. Functional Elbow Range of Motion in Children and Adolescents. J. Pediatr. Orthop. 2020, 40, 304-309. [CrossRef]

24. Borsa, P.A.; Laudner, K.G.; Sauers, E.L. Mobility and stability adaptations in the shoulder of the overhead athlete. Sports Med. 2008, 38, 17-36. [CrossRef] [PubMed]

25. Carter, A.B.; Kaminski, T.W.; Douex, A.T.; Knight, C.A.; Richards, J.G. Effects of highvolume upper extremity plyometric training on throwing velocity and functional strength ratios of the shoulder rotators in collegiate baseball players. J. Strength Cond. Res. 2007, 21, 208-215. [CrossRef]

26. Byram, I.; Bushnell, B.; Dugger, K.; Charron, K.; Harrell, F.J.; Noonan, T. Preseason shoulder strength measurements in professional baseball pitchers: Identifying players at risk for injury. Am. J. Sports Med. 2010, 38, 1375-1382. [CrossRef] [PubMed]

27. Jayanthi, N.; Pinkham, C.; Dugas, L.; Patrick, B.; Labella, C. Sports specialization in young athletes: Evidence-based recommendations. Sports Health 2013, 5, 251-257. [CrossRef] [PubMed]

28. Shank, J.R.; Singleton, S.B.; Braun, S.; Kissenberth, M.J.; Ramappa, A.; Ellis, H.; Torry, M.R. A comparison of forearm supination and elbow flexion strength in patients with long head of the biceps tenotomy or tenodesis. J. Arthrosc. Relat. Surg. 2011, 27, 9-16. [CrossRef]

29. Ellenbecker, T.S.; Davies, G.J. The application of isokinetics in testing and rehabilitation of the shoulder complex. J. Athl. Train. 2000, 35, 338-350.

30. Mayer, F.; Horstmann, T.; Bäurle, W.; Grau, S.; Handel, M.; Dickhuth, H.H. Diagnostics with isokinetic devices in shoulder measurements potentials and limits. Isokinet. Exerc. Sci. 2001, 9, 19-25. [CrossRef]

31. Trakis, J.E.; McHugh, M.P.; Caracciolo, P.A.; Busciacco, L.; Mullaney, M.; Nicholas, S.J. Muscle strength and range of motion in adolescent pitchers with throwing-related pain: Implications for injury prevention. Am. J. Sports Med. 2008, 36, $2173-2178$. [CrossRef] [PubMed]

32. Maly, T.; Zahalka, F.; Mala, L.; Cech, P. The bilateral strength and power asymmetries in untrained boys. Open Med. 2015, 10, 224-232. [CrossRef] [PubMed]

33. Yoshizaki, K.; Hamada, J.; Tamai, K.; Sahara, R.; Fujiwara, T.; Fujimoto, T. Analysis of the scapulohumeral rhythm and electromyography of the shoulder muscles during elevation and lowering: Comparison of dominant and nondominant shoulders. J. Shoulder Elbow. Surg. 2009, 18, 756-763. [CrossRef] [PubMed] 